\title{
LA INTERPRETACIÓN ANTIGUA DE LOS VERSOS LÍRICOS DE HORACIO
}

The relatively reduced number of ancient works related to the metrics of Horace's odes and epodes, as well as the limitted number of metric forms appearing in these poetic works, make possible an extended study of all of them in ancient metrics. This type of study allows, on the one hand, a clear understanding of the devices in ancient metrics; these become clear once they are applied on metrical structures other than the datylic-iambic ones. On the other hand, this type of study may shed some light on Horace's understanding of metrics. This paper presents a detailed description of the interpretation of Horace's lyric metres in ancient artes grammaticae and metricae, and scholia.

El análisis de la versificación de Horacio que llevó a cabo la métrica latina tiene un interés incuestionable desde dos perspectivas distintas: desde la perspectiva de la doctrina métrica antigua y desde la perspectiva de la obra de Horacio.

La interpretación que la métrica latina hizo de la versificación de Horacio, y en concreto de su versificación lírica, constituye una muestra excelente para entender los principios por los que se rige dicha métrica. Por ejemplo, el principio de que los versos originarios fueron el hexámetro dactílico y el trímetro yámbico y de que todos los demás eran derivados de alguno de ellos o resultado de la unión de commata aislados de uno y otro es lógico que se aplique a versos como los arquiloqueos, efectivamente así constituidos. En cambio, las cosas no son ya tan fáciles cuando se pretende aplicar esos mismos principios a versos como los eólicos, de naturaleza distinta, y, por tanto, esencialmente ajenos a los postulados teóricos según los cuales se quiere analizarlos. Se produce entonces un choque entre la doctrina y la materia a la que dicha doctrina se aplica. $Y$ es ese mismo enfrentamiento el que pone al descubierto facetas interesantes tanto del material que se analiza como de los métodos y principios que se emplean en dicho análisis. Es evidente, por tanto, la rentabilidad de su estudio a la hora de caracterizar la métrica antigua. 
Por lo que se refiere a la obra de Horacio, no se trata sólo de esclarecer cómo los antiguos interpretaron sus versos aplicando los principios de análisis métrico con los que en general operaban; un estudio de esta interpretación, al menos en las manifestaciones tardías de que disponemos, puede contribuir a esclarecer el problema de si el propio Horacio conoció y participó de este análisis.

Pero aquí y ahora nuestro propósito es bastante más modesto; como trabajo preliminar, nos vamos a limitar a describir de una manera exhaustiva y orgánica cómo fueron interpretados los versos líricos de Horacio por parte de los metricólogos latinos. De este modo quedarán sentadas las bases para lo que luego puedan ser nuevos intentos de profundizar en la comprensión tanto de las doctrinas métricas antiguas como de la praxis versificadora de Horacio.

1. Hay que empezar diciendo que es relativamente reducido el número de textos conservados que de una u otra forma explicaron la métrica de las odas y los epodos de Horacio. Esto, y el hecho de que sea limitado el número de formas métricas distintas que incorporan estas composiciones, hace posible estudiar de forma exhaustiva todo el material disponible y permite esperar de dicho estudio unos resultados suficientemente significativos.

1.1. Los escritos latinos que estudian las formas métricas de las odas y epodos de Horacio pueden formar parte de obras de carácter diverso. Además de los tratados monográficos de Ps. Cesio Baso ${ }^{1} \mathrm{o}$ de Servio, lo normal es encontrar un capítulo De metris Horatii incorporado a un tratado de métrica o a la parte de métrica de un tratado de gramática. Es el caso de la mayoría de los estudios antiguos sobre la métrica de Horacio de que disponemos: los capítulos De metris Horatii de Cesio Baso, de Terenciano Mauro o de Atilio Fortunaciano forman parte simplemente de un ars metrica; los de Mario Victorino-Aptonio y Diomedes de la parte de métrica de un tratado de gramática. Por fin, disponemos del comentario a Horacio de Ps. Acron, donde, al tratar

' Por orden alfabético de autores, las referencias de las obras que vamos a utilizar son estudiados son las siguientes: Atilio Fortunaciano, De metris Horatii, H. Keil, Grammatici Latini (GLK) VI, 294-304; Cesio Baso, Fragmentum de metris, GLK VI, 254-272, especialmente, De reliquis Horatii metris, 266-272; Diomedes, De uersuum generibus, GLK I, 508-515 y De metris Horatianis GLK I, 518-529; Mario Victorino, De metris Horatianis, GLK VI, 160-184; Ps. Helenio Acrón, Scholia in Horatium uetustiora, O. Keller, Leipzig 1902; Ps. Baso, De metris Horatii, GLK VI, 305306; Servio Honorato, De metris Horatii ad Fortunatianum, GLK IV, 468-472; Terentiano Mauro, De metris, GLK VI, 364-413. 
cada una de las composiciones horacianas, se habla también de su metro.

1.2. En el marco de las obras más amplias como un ars metrica, incorporada o no a un ars grammatica, conviene precisar exactamente qué parte del tratado ocupa el pasaje sobre los versos de Horacio y qué función tiene en él.

Según la concepción de la métrica que subyace a todos estos tratados, una concepción según la cual una secuencia métrica es analizable en unidades jerárquicas que contienen a las inferiores y son contenidas por las superiores, es lógico que cualquier ars de este tipo comience por las unidades mínimas para, en una progresión, llegar al verso o al poema. En consecuencia, una métrica latina debe de articularse en los siguientes capítulos genéricos: De uoce, De littera, De syllaba, De pedibus, De metris, De poematibus ${ }^{2}$. De hecho, todos los trabajos incorporan, como mínimo, unos capítulos dedicados a la sílaba, a los pies y a los metros ${ }^{3}$.

Por lo que se refiere a la parte De metris, prácticamente ${ }^{4}$ todos los manuales suelen desglosarla en varios capítulos o apartados que, también de una manera genérica, podemos denominar De metris prototypis, De metris deriuatis ${ }^{5}$ y De metris Horatii. Los dos primeros suelen ser apartados fijos de todos estos manuales ${ }^{6}$, aunque en lo que a contenidos y organización de los mismos se refiere no se puede establecer un modelo único. El tercer apartado, en cambio, no es de rigor - no lo incluyen los manuales de Sacerdote o Malio Teodoro--, pero suele presentar una relativa uniformidad de contenidos; la organización de los mismos, como en seguida veremos, difiere de unos a otros.

${ }_{2}$ Evidentemente los capitulos De uoce, De littera y De syllaha pueden aparecer en otro lugar si la métrica va incorporada a un ars gammatica.

${ }^{3}$ Que el ars grammatica de Sacerdote no presente, ni en su parte de gramática propiamente dicha ni en la de métrica, un capitulo dedicado al estudio de la silaba no contradice lo que se acaba de señalar, pues presumiblemente en la primera parte, perdida, de su gramática se hablaba de la sílaba.

4 En el caso de Ps. Cesio Baso, el carácter fragmentario de su tratado no hace posible establecer de manera precisa cómo estaba organizado.

5 En este capítulo, y no necesariamente en un capítulo específico, pueden ser analizados algunos metros de Horacio. Este es el caso de Cesio Baso, el de Terenciano Mauro y el del capítulo De uersuum generihus de Diomedes. La diferencia entre estos dos últimos es que mientras en el primer caso sólo se estudian de forma dispersa algunos versos de los carmina los de los epodos se estudian aparte y al final , en el segundo hay un claro propósito de estudiarlos en bloque por mucho que esto se haga en el marco de un capítulo más general, sobre todo tipo de metros, que, por entendernos, podemos considerar derivados.

- Sólo Malio Teodoro no ofrece un capitulo especifico sobre metros derivados, aunque al final de su tratado hace un resumen teórico de los contenidos de un capitulo de este tipo, cf. GLK VI 600, 17 ss. 
Así, pues, de todo lo que respecto a la organización de un tratado de métrica, en general, y de sus capitulos dedicados a metros, en particular, acabamos de decir podemos concluir que el estudio de los metros de Horacio, si se aborda en un apartado específico, es siempre al final del tratado, una vez estudiados los metra prototypa (fundamentalmente el hexámetro y el trímetro yámbico) y los metros derivados de éstos o formados por combinación de cola de ambos. No en vano, estos capítulos sobre los metros de Horacio vienen a completar, en cierto modo, lo que en el sistema métrico alejandrino eran los metra mixta, inconexa, etc., o en el pergameno los metra quae ex utroque (hexámetro y trímetro yámbico) deriuantur, estudiados en los capítulos precedentes del tratado de métrica.

Pero no sólo hay que resaltar la clara integración de un capitulo como éste en la estructura general de los tratados antiguos; es preciso, sobre todo, poner de relieve la peculiaridad que, en virtud del mismo, presentan los manuales latinos con respecto a lo que era el esquema de un manual de métrica griego; con las dos consecuencias que de ello pueden extraerse. En primer lugar, la constatación del especial significado de Horacio como poeta; lo que se refleja, no ya en su repercusión en la versificación y poesía posterior, sino en el hecho, en gran medida relacionado con el anterior, de que la escuela se ocupara sistemáticamente de él.

En segundo lugar, el hecho de que esta novedad de los tratados latinos podría apoyar la idea de algunos de que Horacio hubiera participado ya de la corriente de interpretación métrica pergameno-varroniana que por entonces empezaba a consolidarse en Roma y que, precisamente, a partir de Horacio y gracias a sus intérpretes e imitadores se asentaría en seguida tanto en la teoría (Baso) como en la práctica (Séneca).

1.3. Cuando en un tratado aparece un capitulo especifico sobre los metros de Horacio, dicho capítulo puede aparecer estructurado de dos formas distintas: o bien se pasa revista a todas las odas y epodos, como es el caso de Diomedes, o bien se atiende sólo a las odas o epodos en que un metro aparece por primera vez, quia ille fidicen uno metri genere pluribus saepe utitur cantibus, satis erit semel ratione reddita ceterarum similitudinem primis uersibus denotare (Servio, GLK IV 468, 8-10). En este último caso, ello se puede hacer con mayor o menor lujo de detalles: Atilio Fortunaciano lo hace sin más especificaciones; Mario Victorino, en su primera exposición, añade simplemente el número de veces que aparece el verso en las odas; en su segunda exposición, en cambio, añade la relación completa de odas y epodos escritas en el metro en 
cuestión, identificándolas mediante el número de la oda y el libro en que aparece y el primer verso de la composición. De la misma forma, Servio proporciona también la relación completa de odas que han sido escritas con un mismo metro, aunque las identifica solamente mediante la cita de su primer verso.

Pero ya hemos visto que había casos en que los metros de Horacio no se estudiaban en un capítulo específico de una métrica o de una gramática, sino en un capítulo más general, y que había casos en que la métrica de Horacio era un aspecto más del comentario a su obra. Tratamientos como los del primer tipo, al estudiar los metros de Horacio en el marco más amplio de un capítulo sobre metros compuestos o derivados, no pueden atender a los distintos metros según el orden en que aparecen en su obra sino una vez que se ha hablado de aquellos de los que derivan; todo lo contrario del tratamiento de un comentario, como el de Ps. Acrón, que lógicamente se organiza analizando composición por composición, y caracterizado, en consecuencia, por contemplar la versificación de cada oda en el orden en que éstas aparecen en la edición que maneja.

Tomando en consideración todo lo que acabamos de decir sobre los diferentes procedimientos seguidos a la hora de estudiar los metros de Horacio y el diferente carácter de las obras en que ello se hace, es fácil apreciar que entre las dos posiciones que representan Baso, Terenciano Mauro y Diomedes en De uersuum generibus, por un lado, y Ps. Acrón, por otro, las de Atilio Fortunaciano, Servio, Mario Victorino o Diomedes en De metris Horatianis, aunque en distinta medida, están más próximas a Ps. Acrón ${ }^{7}$. Dicho de otra forma, probablemente el origen de estos tratados haya que buscarlo en comentarios del tipo de aquél al que remonta el de Ps. Acrón, lo que explicaría el orden de exposición que siguen, sin olvidar que ya el propio Horacio parece haber tenido la preocupación de presentar al comienzo de su primer libro de odas lo más significativo del material que luego iba a seguir empleando, con todo lo que ello puede significar de conciencia teórica y de actitud programática previa por parte del poeta.

7 Tan evidente es esto en el caso de Diomedes, que, como Ps. Acrón, no sólo analiza oda por oda y epodo por epodo, sino que al final también trata especificamente el Carmen Saeculare, lo que en un tratado de métrica era completamente gratuito. habida cuenta de que su estructura métrica ya había sido sobradamente estudiada a propósito de otras composiciones. A reforzar esta idea de la estrecha relación entre Diomedes y Ps. Acrón viene, además, la proximidad entre las interpretaciones del gramático y del escoliasta. 
2. Una vez apuntado lo que sobre el sentido y alcance de estos $D e$ metris Horatii dentro de la teoría métrica antigua puede sugerir tanto su propia ubicación en la estructura general de los tratados donde aparecen como su organización interna, podemos dar ya el siguiente paso en nuestro intento de determinar cómo entendieron los antiguos cada uno de estos metros.

2.1. Antes que nada es necesario establecer cuáles son exactamente los metros que vamos a considerar. En realidad, como acabamos de decir, ya el propio Horacio parece haber dispuesto al principio de la obra, intencionadamente y de manera programática, aquellas composiciones cuyos metros iba luego a utilizar repetidamente a lo largo de la misma. El autor antiguo que se disponía a estudiar estos metros no tenía, pues, más que recorrer las odas que encabezan el libro primero para dar cuenta de casi todas las demás.

En efecto, incluso tratamientos como el de Diomedes o el de Ps. Acrón se limitan, prácticamente, a explicar los metros que aparecen en las primeras odas para luego hacer referencia a la explicación alli ofrecida. Atilio Fortunaciano, Mario Victoriano o Servio lo hacen de una forma más estricta, es decir, se limitan a estudiar, respectivamente, las once menos la décima-, trece - menos la duodécima- y diez primeras odas ${ }^{8}$.

${ }^{8}$ Que Atilio Fortunaciano se ocupe sólo de las once primeras odas, excluyendo la décima, Mercuri facunde nepos Atlantis, significa claramente que se limita a analizar cada metro la primera vez que aparece y que considera, por tanto, que la oda décima no incorpora un metro distinto de la segunda, Iam satis terris niuis atque dirae, ya analizada como una composición de tres endecasílabos sáficos y un adonio. Esto, sin embargo, no parece coincidir con la propia interpretación de Horacio que, al incluirla en esta serie inicial de odas, en la que la undécima incorpora evidentemente un metro distinto a los de las anteriores, parece haber concebido la composición como distinta de la segunda: efectivamente Mercuri facunde nepos Allantis presenta en su primer sáfico fin de palabra en la sexta silaba frente al corte habitual en quinta que presentan los sáficos de otras odas.

La postura de Mario Victorino, en cambio, es algo ambigua, porque, siendo su relación de odas igual a la de Fortunaciano, si se detiene en la oda $X$, si bien es verdad

a) que al explicarla remite a la segunda,

b) que en realidad su exposición incluye también la oda XIII, cuyo metro ya habia aprecido en la oda III y

c) que, en su segunda exposición, en la relación de las composiciones que incorporan cada metro incluye este caso en la lista de odas con endecasílabos sáficos, que viene encabezada, y esto es significativo, por la oda II.

Se podria explicar esta postura de Mario Victorino diciendo simplemente que el autor ha procedido a una revisión algo mecánica de las primeras odas, incluyendo entre ellas la X pero también la XIII, en una postura a caballo entre la de los comentaristas de Horacio y Atilio Fortunaciano. No es ésta, sin embargo, una explicación que cuadre a un texto tan minucioso como el de Mario Victorino; por ello nos 
Pero, si estos tratadistas querían ser exhaustivos, tenían que recoger necesariamente otros metros que aparecen más adelante y no figuran entre los diez u once primeros carmina, además de describir los que el poeta emplea en los epodos. Se trata de Non ebur neque aureum (C. II 18), Miserarum est neque amori dare ludum (C. III 12) y Diffugere niues redeunt iam gramina campis (C. IV 7) ${ }^{9}$.

Según esto el corpus de versos horacianos cuya interpretación antigua nos interesa estaría formado por los que figuran en las once primeras odas del libro primero más los tres que acabamos de citar más aquellos en que fueron escritos los epodos. Tenemos, que hacer, sin embargo, dos restricciones. En primer lugar, hablando de una manera rigurosa, no todos los versos de estas composiciones son nuevos: algunos de ellos pueden haber intervenido en otras estrofas anteriormente analizadas. Es por eso por lo que parece mejor hablar de versos que de estrofas. En este sentido, Mario Victorino facilita una relación con todos los versos distintos utilizados por Horacio. Adjuntamos, además, la composición de la que forman parte y el nombre con que vamos a identificar el verso en nuestro análisis.

1. Maecenas atauis edite regibus (C. I 1), asclepiadeo.

2. Iam satis terris niuis atque dirae (C. I 2), sáfico.

3. Terruit urbem (C.I 2), adonio.

4. Sic te diua potens Cypri (C. I 3), gliconio.

5. Tu ne quaesieris, scire nefas, quem mihi, quem tibi (C. I 11), asclepiadeo mayor.

6. Soluitur acris hiems grata uice ueris et Fauoni (C. I 4), $1 .^{\mathrm{er}}$ verso de la estrofa arquiloquea IV.

7. Trahuntque siccas machinae carinas $(C$. I 4,2$), 2 .^{\circ}$ verso de la estrofa arquiloquea IV, trímetro yámbico cataléctico.

8. Grato, Pyrra, sub antro $(C$. I 5, 3), ferecracio.

9. Laudabunt alii clara Rhodon aut Mytilenen ( $C$. I 7), $1{ }^{\text {er }}$ verso de la estrofa alcmania, hexámetro dactílico.

10. Aut Epheson bimarisue Corinthi $(C .17,2), 2 .^{\circ}$ verso de la estrofa alcmania, tetrámetro dactilico.

parece más acertado pensar que la incorporación de las odas X y XIII, precisamente, puede responder a una intención de ofrecer un ejemplo también de los primeros casos en que un metro se repite por primera vez. En este sentido estaría completamente justificada la omisión de la oda XII, que incorpora el mismo metro que la X (y que la II), un metro, por tanto, que se repite por segunda vez.

$\checkmark$ Hay que observar que el orden de odas de la edición de Wickham y Garrod no coincide siempre con el ofrecido por los tratadistas latinos ni el de éstos entre sí. Asi C.II 18 es en Mario Victorino C.II 16 y en Diomedes C.II 17 y C. III 12 es C.III 7 en Diomedes. 
11. Lydia, dic, per omnis (C. I 8), aristofanio.

12. Hoc deos vere, Sybarin cur properes amando $(C$. I 8, 2), sáfico mayor.

13. Vides ut alta stet niue candidum (C. I 9), endecasílabo alcaico.

14. Siluae laborantes, geluque $(C$. I 9, 3), eneasílabo alcaico.

15. Flumina constiterint acuto $(C$. I 9, 4), decasílabo alcaico.

16. Non ebur neque aureum (C. II 18), 1 . $^{\text {er }}$ verso de la estrofa hiponactea.

17. Mea renidet in domo lacunar $(C$. II 18,2$), 2 .^{\circ}$ verso de la estrofa hiponactea, trímetro yámbico cataléctico.

18. Ibis Liburnis inter alta nauium $(E p .1,1)$, trímetro yámbico.

19. Amice, propugnacula $(E p .1,2)$, dímetro yámbico.

20. Scribere uersiculos amore percussum graui (Ep. 11, 2), elegiambo.

21. Niuesque deducunt Iouem; nunc mare, nunc siluae (Ep. 13, 2), yambélego ${ }^{10}$.

${ }^{10} \mathrm{Si}$ se atiende a la relación de odas a las que pertenecen los versos seleccionados, se observará, en primer lugar, que el verso número 5 , un Asclepiadeo mayor, es el primero de la oda XI por lo que debía haber sido recogido tras el verso número 15, perteneciente a la oda IX, la última de las del libro I contemplada (en realidad este verso quedaba descolgado de la relación al no considerarse el endecasílabo sáfico de la oda $X$ ).

En segundo lugar, que no aparece recogido verso alguno de C.I 6, de C.III 12 y de $C$.IV 7, así como de los epodos 14, 16 y 17 (estos tres últimos los comenta incluso Atilio Fortunaciano en una exposición limitada exclusivamente a dar cuenta de aquellos epodos que presentan una forma métrica nueva, y no de todos, como hacen otros tratadistas). La razón de que se excluyan todos estos versos es justamente que ya habian aparecido en otras combinaciones estróficas y que, por tanto, ya habian sido comentados. La ausencia en la relación de la oda XII del libro III, escrita en jónicos, está justificada porque de metros jónicos ya había hablado el autor cuando trató de los prototypa (89-92) y porque se trata, en definitiva, de versos de naturaleza distinta a la de los que aquí se contemplan.

En tercer lugar, se observará que tanto el verso 7 como el 17 son trímetros yámbicos catalécticos, con la inconsecuencia que esto supone en una relación exclusivamente formada por todos los versos distintos utilizados por Horacio. La explicación de este aparente error del autor puede orientarse en el sentido de que, si como verso aislado el verso 17, no constituye una estructura métrica distinta de la del verso 7 , unido al verso 16, con el que forma estrofa, sí constituye una combinación por primera, y única vez, utilizada por Horacio. Hay en el fondo de todo ello una confusión en la utilización y empleo del término metrum, frecuente en la métrica latina, según la cual metrum puede significar tanto uno de los versos de una combinación estrófica como la propia estrofa. Así, pues, el "metro» 17, entendiendo metro como verso ya ha sido computado antes, con lo que el autor tenía que haber prescindido de él en su relación; pero entendiendo metro como estrofa de la que este verso forma parte, es la primera vez que aparece con lo que hasta cierto punto podría estar justificado que el autor se ocupe de él.

Servio analiza prácticamente los mismos versos incluidos en la relación de Mario Victorino. Que, sin embargo, sean diecinueve los metros que estudia se debe a que, 
Además de la anterior, creemos conveniente hacer una nueva restricción, esta vez sobre la relación de versos de Mario Victorino. En efecto, no parece muy interesante estudiar la interpretación antigua de los versos 9 y 10 ó 19 a 21 , cuyo carácter dactílico, yámbico o dactílico-yámbico estaba fuera de toda duda. Tampoco lo parece la de algunas otras formas líricas jonias, como los versos 6 y 7, por un lado, y 16 y 17, por otro, que, aunque de estructura algo más compleja, no suscitaban la diversidad de interpretaciones que suscitaba prácticamente toda la lírica eólica horaciana"

2.2. No es éste el lugar - ni parece necesario - de ofrecer una a una todas las interpretaciones de cada uno de los versos que vamos a tomar en consideración en cada una de las, a veces dobles, exposiciones de cada autor, por mucho que en la base de todas nuestras consideraciones esté necesariamente el minucioso trabajo previo de este tipo que hemos tenido que llevar a cabo para poder hacerlas aqui y ahora. Resultaría igualmente largo y hasta cierto punto inútil exponer uno a uno los datos derivados de combinar todos los anteriores, referentes a los autores y sus interpretaciones concretas de cada metro, con una nueva variable, a saber, la distinta interpretación genérica del metro que cada interpretación concreta de cada verso puede suponer en cada autor.

En efecto, según hemos concluido en otra ocasión ${ }^{12}$, un mismo verso de Horacio puede ser interpretado bien como una estructura coriámbica bien como una estructura dactílico-yámbica, derivada, por tanto, del hexámetro dactílico y/o del trímetro yámbico (considerando o no la catalexis como recurso interpretativo), interpretación esta última que no excluye el reconocimiento de componentes trocaicos, dada la intima relación entre yambo y troqueo. Además, es posible que el verso sea interpretado como directamente derivado de otro verso, a su vez anteriormente interpretado como dactílico-yámbico; o que el verso sea simplemente interpretado por pies, en sucesión arbitraria, de forma que resulta dificil suponer en esta secuencia de pies una escansión dactílicoyámbica o coriámbica implícitas.

Según esto, para la métrica antigua, prescindiendo de estas últimas

no sin cierta lógica, ha prescindido de hablar del adonio y del Sáfico mayor y, lo que es más interesante, del comprometido verso 17 , en cuyo lugar habla de los jónicos, que echábamos de menos en Mario Victorino.

"Quede claro que en la lirica eólica horaciana hay versos que, como veremos, tienen una interpretación exclusivamente dactílico-yámbica en la métrica antigua. Aun así, no alcanzan nunca una explicación tan univoca como la que puedan tener los versos 9 y 10 y los de los epodos.

12 La métrica latina en el siglo IV. Diomedes y' su entorno, Granada 1990, pp. 213-214. 
escansiones por pies, más bien mecánicas, cabría distinguir tres tipos de versos horacianos:

a) aquellos que pueden tener una interpretación dactílico-yámbica pero también coriámbica;

b) aquellos que tienen una interpretación exclusivamente dactílicoyámbica en alguno de los sentidos que acabamos de decir;

c) aquellos que tienen una interpretación exclusivamente coriámbica.

2.2.1. Son versos susceptibles de recibir tanto una interpretación dactílico-yámbica como coriámbica el asclepiadeo y el asclepiadeo mayor, el sáfico, y el gliconio y el ferecracio.

2.2.1.1. En el caso del asclepiadeo, hay autores que ofrecen una interpretación dactílica, pudiéndose añadir la coriámbica o no - este último es el caso de Diomedes en sus dos exposiciones-. La interpretación dactílica más común es la que considera el asclepiadeo como un metro derivado del pentámetro, así Diomedes en De uersuum generibus (508, 5-11): Asclepiadeum ab auctore dictum, cuius exemplum est Maecenas atauis edite regibus. his potest, unde ortus est, ad pentametrum elegiacum redigi addita una syllaba sic, Maecenas atauis edite remigibus. quod tale est quale illud supra, candida caeruleo nata Venus pelago.

Pero también encontramos la interpretación que se esfuerza en explicarlo como directamente derivado del hexámetro. El propio Diomedes en el mismo capitulo añade (508, 11-21): potest Asclepiadeus ab hexametro nasci detracto in mediis partibus disyllabo verbo et in ultimis, ut si dicas nimborum in patriam, loca feta furentibus Austris, nimborum in patriam feta furentibus... rursus illi Asclepiadeo adde disyllabum uerbum in medio et in imo, facies hexametrum sic, Maecenas atauis ades edite regibus olim.

Sin embargo, existen exposiciones que prefieren empezar por la explicación coriámbica, pudiendo ofrecer o no la dactílica. Nos referimos a la de Baso ${ }^{13}$, Ps. Baso y Mario Victorino en su pasaje [De numero eorum], a Servio y a Ps. Acrón. En realidad, se suele tratar de un análisis por pies entre los que se distingue el coriambo, lo que implícitamente

${ }^{13}$ Es necesario aclarar que Baso habia discutido la escansión de este verso en un pasaje perdido de su obra. No obstante, al comentar otros versos relacionados con el asclepiadeo hace una serie de referencias a la interpretación que habia hecho del mismo; de ellas podemos colegir que no sólo optaba por la interpretación coriámbica, sino que se manifestaba abiertamente contra la dactilica: Maecenas atauis edite regibus. sed qui altius haec non perpexerunt grammatici hoc putant metrum de curtato pentametro facto, ut reddita syllaba fiat [pentametrum] tale, Maecenas atauis edite remigibus" $(268,23-26)$. 
supone una interpretación coriámbica: Prima igitur ode monocolos est. nam uersus, qui asclepiadeus dicitur, constat spondius, duobus choriambis et pyrrichio, Servio $(468,16-17)$. Pero esta explicación coriámbica es todavía más explícita cuando en ella se alude expresamente a los metros coriámbicos del verso: quidam uolunt adiectis duobus pedibus, spondio ad caput, pyrrichio ad finem, in medio dimetron choriambicon clausum, ut ingressio sit talis, Maecenas atauis edite re,gibus. nam si solas syzygias pronunties, choriambicum sonant, Atilio Fortunaciano (296, 16-19). Habremos de repetir las mismas observaciones cuando hablemos de los metros exclusivamente interpretados como coriámbicos.

Otras dos posibles interpretaciones completan la visión que de este controvertido verso tenía la métrica latina. Nos referimos a aquella que lo considera como un endecasílabo falecio más una sílaba, lo que implica, por tanto, una interpretación dactílico-yámbica del mismo, ya que del falecio pars prior de hexametro est... posterior autem pars de principio iambici est, Diomedes $(509,14-16)^{14}$.

La segunda explicación a que nos referíamos es la que, ofrecida por Atilio Fortunaciano o por Ps. Baso, considera el verso como un trímetro antispástico: potest et antispasticon esse trimetron acatalecton, cuius paenultimam affectauit pro longa breuem facere, cuius ingressio est talis, Maecenas a,tauis edi,te regibus, Atilio Fortunaciano (206, 10-13).

En cuanto al asclepiadeo mayor, hay tratadistas a los que probablemente por el carácter más conciso de su exposición, debió de parecerles innecesario detenerse a comentar su estructura, una vez que se había hablado de la estructura del asclepiadeo. Es el caso de Ps. Baso, Atilio Fortunaciano, Diomedes en De uersuum generibus y Servio.

Otros, en cambio, viendo en éste un verso distinto del primero, no pudieron dejar de describirlo de la misma manera que habían descrito tanto el sáfico como el sáfico mayor. De entre ellos hay quienes explican detenidamente en qué consiste la relación de este verso con el asclepiadeo e incluso con el gliconio: quale est illud apud Horatium, quod et ipsum ab Alcaeo sumpsit, tu ne quaesieris, scire nefas, quem mihi quem tibi. hoc ipsum imminuendo paulatim tria haec metra quae rettuli manifestiora faciam, tu ne quaesieris quem mihi quem tibi: hoc par est illi, Maecenas atauis edite regibus. alterum detraham choriambum, fiet tu ne quaesieris tibi: hoc par est illi, intermissa Venus diu et castae Pierides meae,

${ }^{14}$ No es extraño que en la métrica antigua se establezca una relación entre el asclepiadeo y el falecio, aparentemente sólo comparables en sus seis primeras silabas

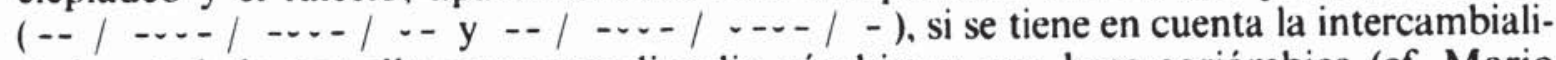
dad postulada por ella entre una dipodia yámbica y una base coriámbica (cf. Mario Victorino, 90, 10-13 y 93, 6-94, 6, por ejemplo). 
Cesio Baso $(259,30-260,11)$. De una forma menos minuciosa, también Mario Victorino establece la relación entre ambos al describir el asclepiadeo mayor como un asclepiadeo al que se ha añadido un coriambo, aunque el resultado, en opinión de algunos, pueda interpretarse como un metro antispástico ${ }^{15}$.

En cierto modo, también queda expresada la relación de ambas estructuras métricas cuando se describe el asclepiadeo mayor exactamente igual que un asclepiadeo pero, lógicamente, con tres coriambos en lugar de con dos, tal como hacen Mario Victorino en [De numero eorum], Diomedes, aunque no la primera vez que describe el metro, o Ps. Acrón. Dice, por ejemplo, Diomedes: octaua decima ode Sapphicum metrum heccedecasyllabum habet, quod constat ex spondeo et tribus choriambis et pyrrichio $(552,4-5)$.

Por fin, que Diomedes, la primera vez que describe el metro, y Ps. Acrón llamen a este verso Phallaecium metrum supone también ponerlo en relación con el asclepiadeo. Ya hemos visto que, al menos Diomedes, explicaba el asclepiadeo como derivado del endecasílabo falecio y que el endecasílabo falecio se interpretaba como una estructura métrica formada por un comma del hexámetro y otro del trímetro yámbico. Ello implica, por tanto, que también el asclepiadeo mayor se interpretaba como una estructura dactílico-yámbica. Quizá de esta forma haya que entender la aparentemente simplista escansión por pies que acompaña en ambos tratadistas a este denominación del verso: Metrum choriambicum exdecasyllabum, quod constat ex spondeo, tribus choriambis et pyrrichio sive iambo; ita: tu ne • quaesieris • scire nefas • quem mihi quem • tibi. siue aliter: metrum Fallecium, quod scanditur ita: tu ne $\bullet$ spondeus $\bullet$ quaesie $\bullet$ dactilus $\bullet$ ris $\bullet$ semipes $\bullet$ scire ne $\bullet$ dactilus $\bullet$ fas $\bullet$ semipes $\bullet$ quem mihi • dactilus • quem tibi • dactilus (I, p. 53).

2.2.1.2. Aunque también el sáfico recibe tanto lo que hemos dado en llamar interpretaciones dactílico-yámbicas como interpretaciones coriámbicas, ninguna de ellas presenta la variedad de escansiones que acabamos de ver para el asclepiadeo. En efecto, en lo que se refiere al primer tipo de interpretación, los tratadistas no sólo suelen coincidir en la escansión del verso sino también en ofrecerla en primer lugar: hos hendecasyllabos alii alcaicos, alii sapphicos uocant prima in his caesura trochaica est: nam habet dimetron brachycatalecton, sequens iambica dimetron hypercatalecton, Atilio Fortunaciano (297, 1-3). Añadamos que

15 Es curioso que el autor, en cambio, al hablar del Asclepiadeo no haya of recido la posible interpretación antispástica del mismo que vimos en Atilio Fortunaciano. 
también es posible encontrar una explicación más "rudimentaria», es decir, una explicación que no utiliza el concepto de catalexis: superior pars ex trochaico est. nam si haec uerba «iam satis terris» suppleas facies integrum trochaicum sic, iam satis terris uirente secta pinus in Crago. inferior autem, uerba haec "niuis atque dirae», de principio iambici sunt, denique additis quae desunt iambicus poterit inpleri sic, niuis atque dirae secta pinus in Crago, Diomedes $(508,25-30)$. Con todo, no encontramos una explicación de este tipo en la breve relación de metros horacianos del pasaje [De numero eorum] de Mario Victorino o en exposiciones, también concisas, como las de Servio o Ps. Acrón. También Ps. Baso se limita a ofrecer la explicación coriámbica.

La escansión coriámbica, tal como suele darse en este tipo de tratados, es decir, por pies, también podemos registrarla en casi todos los autores. Diomedes en De uersuum generibus, donde se prescinde de forma prácticamente absoluta de explicaciones de este tipo, si se excluye el aristofanio y el sáfico mayor, que reciben en todos los autores explicaciones exclusivamente coriámbicas, constituye una excepción a lo que acabamos de decir. Tampoco Servio habla de esta posibilidad, limitándose a ofrecer una escansión por pies, del tipo de la que ofrece Mario Victorino $(162,23-25)$ feritur hoc metrum uel per simplices pedes, quorum primus trochaeus, secundus spondeus, tertius dactylus, quartus trochaeus, quintus spondeus uel trochaeus, puramente mecánica, y no del otro tipo, también por pies, aunque no mecánica, uel per dipodian ut constet epitrito secundo, quem et hippium dicimus, dehinc choriambo, ultimo bacchio uel amphibrachy; ambas en Mario Victorino (162, 25-26), a continuación, por tanto, de la anterior.

En la línea de las interpretaciones dactílico-yámbicas no podemos dejar de hablar de la que propone Atilio Fortunaciano. Considera este autor el endecasílabo sáfico como un endecasílabo alcaico, a su vez interpretado siempre en la métrica antigua como derivado del hexámetro y de trímetro yámbico, cuya primera sílaba ha pasado al final ${ }^{16}$ : nasci autem uidetur ab alcaico hendecasyllabo... huius si primam syllabam in ultimum transtuleris, facies hendecasyllabum sapphicum (297, 9-14).

2.2.1.3. El gliconio (y también el ferecracio) participa igualmente de ambos tipos de interpretación, la dactílico-yámbica y la coriámbica. Predomina la dactílica: Tertia ode dicolos est distrofos. nam primus versus, cui glyconio uocabulum datur, constat spondio et duobus dactylis, Servio (468, 11-12).

${ }^{16}$ Puede apoyar esta interpretación el hecho de que otros autores vacilen entre atribuir el verso a Safo o a Alceo. 
Pero también encontramos interpretaciones coriámbicas en Mario Victorino y en el capitulo De metris Horatianis de Diomedes: tertia ode dicolos Horatianum metrum habet, cuius prior uersus constat Glyconius et recipit tres pedes, spondeum choriambum iambum, Diomedes (519, 2123).

Por fin, también para este metro, vuelve a considerarse la posibilidad de relacionarlo con otro, que puede ser el asclepiadeo - como sugieren Baso y Ps. Cesio Baso, lo que en estos autores supone una interpretación coriámbica del mismo-, el endecasílabo sáfico, - cosa que, además de Diomedes en De uersuum generibus, hace Mario Victorino-o el Priapeo - como proponen de nuevo Baso y Atilio Fortunaciano.

2.2.2. Exclusivamente dáctilo-yámbico es, además del del adonio, el análisis que hacen todos los autores del endecasílabo, eneasílabo y decasílabo alcaico.

2.2.2.1. Respecto al endecasílabo, en todos los autores lo encontramos interpretado como combinación de una comma del hexámetro y otro del trímetro yámbico. Valga como ejemplo la descripción de Diomedes en De metris Horatianis (520, 28-521, 3): nona ode metrum Alcaicum habet et scanditur per quaternos uersus... primus et secundus uersus ex penthemimere constat iambico et duobus dactylis.

También las de Atilio Fortunaciano y Diomedes en De uersuum generibus son interpretaciones de este tipo, aunque contemplen el verso como la combinación de una parte del trímetro yámbico y otra del asclepiadeo. Recordemos que una de las posibles interpretaciones del asclepiadeo era la dactílica, considerándolo como derivado del hexámetro, bien directamente, bien indirectamente, a través del pentámetro. No obstante, el asclepiadeo podia también ser entendido como un metro coriámbico de manera que, cuando Baso interpreta el verso como la unión de dos commata, uno del trímetro yámbico y otro del asclepiadeo, su interpretación no es exactamente yámbico-dactílica sino yámbico-coriámbica: constant singula ex duobus commatibus, quorum quod antecedit ex trimetro iambico sumptum est et cum eo compositum sic, uides ut alta genibus haerebo tuis. sequitur comma «stet niue candidum»: ex hemistichio pentametri heroi delibata syllaba compositum est... potest etiam uideri pars metri illius extrema, quod est tale, Maecenas atauis edite regibus, de quo iam disputauimus, ut hoc ita cum illo iungatur, Maecenas atauis stet alta niue candidum. constat enim hoc comma ex choriambo... et iambo $(268,10-21)$.

Atilio Fortunaciano, además, ofrece una explicación exclusivamente yámbica $(301,22-23)$ : alii hendecasyllabos appellant factos ex iambico 
trimetro detractione unius syllaba, quam si restitues, facies trimetro acatalecton.

2.2.2.2. Más simple resulta la interpretación del eneasílabo alcaico entre estos tratadistas. Todos coinciden en que se trata de un metro yámbico, especifiquen expresamente o no que además es cataléctico. Así, como ejemplo del primer tipo de interpretación, leemos en Mario Victorino (166, 20-25): tertius autem erit dimeter cum semipede in syllaba ita, siluae laborantes geluque: trimeter iambicus fiet, si addicias «frigido». nam semipede minor est ab iambico, qui amphimacri adiectione completus est. Y sin necesidad de una explicación tan compleja, por incorporar el concepto de catalexis, en Atilio Fortunaciano (301, 27-302, 1): tertium colon trimetron iambicon hypercatalecton: nam additis tribus syllabis fiet trimetron acatalecton, siluae laborantes geluque candido.

2.2.2.3. También en el caso del decasílabo alcaico todos los autores coinciden en la consideración del mismo como metro dactílico. Sin embargo, dentro de esta perspectiva general, el verso puede ser enfocado de distinta manera. Hay interpretaciones puramente dactílicas: quartum compositum est ex penthemimeri et bacchio. hexametrum de hoc facies redditis quibuscumque partibus sic, flumina constiterint scopulo pugnantia acuto, Atilio Fortunaciano (302, 2-4)], e interpretaciones que pueden ser precisadas mediante el concepto de la catalexis: trimetris quoque epicis hypercatalecticis, ut est hic, flumina constiterint acuto, usus est libro primo septies, secundo decies, tertio undecies, quarto ter, Mario Victorino $(167,7-9)$.

No deja tampoco de ser dactilica una explicación alternativa de Baso que considera el metro derivado del arquebulio, de estructura anapéstica, $y$, por lo mismo, relacionado con el hexámetro.

Pero hay otras interpretaciones que conciben el verso, no como puramente dactílico, sino como dactílico-trocaico. Puede ilustrarlo, por ejemplo, Servio (470, 13-16): Nona ode dicolos est tetrastrofos... quartus autem dactylis duobus et duobus trochaeis terminatur.

2.2.3. Es digna de observación la absoluta unanimidad de todos los autores a la hora de interpretar el aristofanio y el sáfico mayor. Incluso un planteamiento tan reticente a una interpretación de este tipo como el de Diomedes en De uersuum generibus no ofrece otro análisis que el coriámbico para el sáfico mayor y por ende para el aristofanio que explícitamente se pone en relación con el anterior.

De todas formas, como hemos adelantado arriba, hay que distinguir dos tipos de interpretaciones coriámbicas para estos versos. Por un lado, es común ofrecer una interpretación por pies, que implicitamente 
supone una escansión coriámbica, distinta, por tanto, de una escansión mecánica en pies simples. De este último modo escanden todos los autores, excepto Atilio Fortunaciano. Dice así Mario Victorino, por ejemplo: Lydia, dic per omnes / hoc deos vere, Sybarin cur properas amando. Prior uersus, ut diximus ex choriambo et bacchio a breui incipiet, sequens e tribus choriambis adaeque claudente uersum bacchio subsistunt (165, 27-30). La precisa interpretación de Atilio Fortunaciano en los dos casos es, en cambio: primum colon dimetron catalecticun est... secundun colon tetrametrum catalecticum (300, 21-30).

Marina del Castillo 